\title{
Optical soliton perturbation with spatio-temporal dispersion having Kerr law nonlinearity by the variational iteration method
}

\author{
O. González-Gaxiola ${ }^{a, *}$, A. Biswas ${ }^{b, c, d, e}$, A. Kamis Alzahrani ${ }^{c}$, and M. R. Belic ${ }^{f}$ \\ ${ }^{a}$ Departamento de Matemáticas Aplicadas y Sistemas, Universidad Autónoma Metropolitana-Cuajimalpa, \\ Vasco de Quiroga 4871, 05348 Mexico City, Mexico. \\ *ogonzalez@correo.cua.uam.mx \\ ${ }^{b}$ Department of Physics, Chemistry and Mathematics, \\ Alabama A\& M University, Normal, AL 35762-7500, USA. \\ ${ }^{c}$ Department of Mathematics, King Abdulaziz University, Jeddah-21589, Saudi Arabia. \\ ${ }^{d}$ Department of Applied Mathematics, National Research Nuclear University, \\ 31 Kashirskoe Shosse, Moscow-115409, Russian Federation. \\ ${ }^{e}$ Department of Mathematics and Statistics, Tshwane University of Technology, Pretoria-0008, South Africa. \\ ${ }^{f}$ Science Program, Texas A\& M University at Qatar, PO Box 23874, Doha, Qatar.
}

Received 16 January 2020; accepted 1 March 2020

\begin{abstract}
This paper studies optical soliton perturbation that appears with Kerr law nonlinearity having spatio-temporal dispersion. The numerical scheme adopted is the variational iteration method. The perturbation terms are of Hamiltonian type and stem from inter-modal dispersion, self-steepening and nonlinear dispersion. Both bright and dark solitons are taken into consideration.
\end{abstract}

Keywords: Nonlinear Schrödinger equation; Kerr law nonlinearity; variational iteration method; optical solitons solutions; perturbation.

PACS: 02.30.Jr; 02.60.Cb; 45.10.Db; 44.05.+e

\section{Introduction}

The study of optical soliton perturbation has been going on for several decades now [1-4]. This has led to the visibility of several interesting results both from analytical and numerical perspective. While there exists an abundance of analytical tools to handle the governing equations, there exists only handful few numerical schemes that are applicable and are visible. These range from finite element method, finite difference method, Adomian decomposition scheme, LaplaceAdomian decomposition, and the variational iteration method (VIM). This paper implements VIM to address soliton dynamics, modeled by the nonlinear Schrödinger's equation (NLSE), with a few perturbative effects. These perturbation terms come from intermodal dispersion, self-steepening effect, and nonlinear dispersion. The NLSE also includes the effect of spatio-temporal dispersion (STD) in addition to the usual chromatic dispersion (CD). The inclusion of STD, in addition to $\mathrm{CD}$, makes the model well-posed. Both bright and dark solitons will be studied in the current work. The surface plot, contour plot, and the error plots are all presented for both of these solitons. The details of VIM and its application to the model are inked in the rest of the paper.

\section{Governing model}

The dimensionless form of NLSE with STD Kerr law nonlinearity is given by [5]

$$
\begin{aligned}
i u_{t}+a u_{x x} & +b u_{x t}+c F\left(|u|^{2}\right) u \\
& =i\left[\alpha u_{x}+\beta\left(|u|^{2} u\right)_{x}+\gamma\left(|u|^{2}\right)_{x} u\right] .
\end{aligned}
$$

DOI: https://doi.org/10.31349/RevMexFis.66.404

In Eq. (1), $u(x, t)$ represents a complex field envelope, and $x$ and $t$ are spatial and temporal variables, respectively. Here, the coefficient $a$ is the group velocity dispersion (GVD) and $b$ is the STD the coefficient of $c$ represents the Kerr law nonlinear term that is modeled by the functional $F\left(|u|^{2}\right)=|u|^{2}$. This type of nonlinearity originates when a light wave in an optical fiber is subjected to nonlinear responses [6]. Moreover, in the perturbative term of Eq. (1), the first term represents the inter-modal dispersion (IMD), the second term is the self-steepening effect and finally the last term accounts for another version of nonlinear dispersion (ND). This perturbed NLSE is going to be studied by the VIM in this paper, for Kerr law nonlinearity with fully nonlinear perturbation terms.

\section{Bright optical solitons}

The bright optical soliton solution to Eq. (1) is given by [5]:

$$
u(x, t)=A \operatorname{sech}[B(x-\nu t)] e^{i[-\kappa x+\omega t+\theta]} .
$$

Here, $\nu$ is the soliton velocity, $\kappa$ is the soliton frequency, $\omega$ is the angular velocity, and $\theta$ is the phase center.

The amplitude $A$ of the soliton in this case is given by

$$
A=\sqrt{\frac{2\left(\omega+\alpha \kappa-a \omega \kappa+b \kappa^{2}\right)}{c-\beta \kappa}}
$$

and the width $\mathrm{B}$ of the soliton is given by

$$
B=\sqrt{\frac{\omega+\alpha \kappa-a \omega \kappa+b \kappa^{2}}{b-a \nu}} .
$$


The velocity $\nu$ of the soliton in relation to the coefficients that appear in the Eq. (1) is

$$
\nu=\frac{a \omega-2 b \kappa-\alpha}{1-a \kappa},
$$

and the constraints conditions on the parameters in order to guarantee the existence of the bright solitons are

$$
\begin{aligned}
& (c-\beta \kappa)\left(\omega+\alpha \kappa-a \omega \kappa+b \kappa^{2}\right)>0, \\
& (b-a \nu)\left(\omega+\alpha \kappa-a \omega \kappa+b \kappa^{2}\right)>0, \\
& 3 \beta+2 \gamma=0, \quad \text { and } a \kappa \neq 1 .
\end{aligned}
$$

\section{Dark optical solitons}

The dark optical soliton solution to (1) is given by $[5,6]$ :

$$
u(x, t)=A \tanh [B(x-\nu t)] e^{i[-\kappa x+\omega t+\theta]} .
$$

Here, $\nu$ is the soliton velocity, $\kappa$ is the soliton frequency, $\omega$ is the angular velocity, and $\theta$ is the phase center.

The amplitude $A$ of the soliton is

$$
A=\sqrt{\frac{10 \alpha \kappa+10 b \kappa^{2}-\alpha \kappa+1}{10(c-\beta \kappa)}},
$$

while the inverse width $B$ of the soliton in this case is given by

$$
B=A \sqrt{-\frac{c-\beta \kappa}{2(b-a \nu)}} .
$$

The velocity $\nu$ of the soliton is seen in Eq. (5).

Considered Eqs. (10) and (11), the constraint conditions that guarantees the existence of dark solitons are

$$
\left(10 \alpha \kappa+10 b \kappa^{2}-\alpha \kappa+1\right)(c-\beta \kappa)>0,
$$

and

$$
(b-a \nu)(c-\beta \kappa)<0 .
$$

\section{A brief description of the VIM}

According to the variational iteration method, we consider the following nonlinear partial differential equation:

$$
\left\{\begin{array}{l}
L u(x, t)+R u(x, t)+N u(x, t)=g(x, t), \\
u(x, 0)=u_{0}(x), \quad u_{x}(x, 0)=u_{1}(x) .
\end{array}\right.
$$

where $L=\partial / \partial t, R$ and $N$ are linear and nonlinear operators respectively, and $g(x, t)$ is an inhomogeneous term (or source). The variational iteration method admits the use of the correction functional for Eq. (14) which can be written, for every $n \geq 0$, as

$$
\begin{aligned}
u_{n+1}(x, t) & =u_{n}(x, t)+\int_{0}^{t} \lambda(\xi)\left(L u_{n}(x, \xi)\right. \\
& \left.+R \tilde{u}_{n}(x, \xi)+N \tilde{u}_{n}(x, \xi)-g(x, \xi)\right) d \xi .
\end{aligned}
$$

Where $\lambda(\xi)$ is a general Lagrange multiplier, which can be identified optimally via the variational theory [7-10], the second term on the right is called the correction and $\tilde{u}_{n}$ is considered as a restricted variation, scilicet, $\delta \tilde{u}_{n}=0$.

The stationary conditions for Eq. (15) can be obtained as follows:

$$
\left\{\begin{array}{l}
\lambda^{\prime \prime}(\xi)=0, \\
\left.\lambda(\xi)\right|_{\xi=t}=0, \\
1-\left.\lambda^{\prime}(\xi)\right|_{\xi=t}=0 .
\end{array}\right.
$$

Then, the Lagrange multipliers can be identified as

$$
\lambda(\xi)=\xi-t .
$$

Substituting the found multiplier into Eq. (15) results in the following iteration formula, for every $n \geq 0$ :

$$
\begin{aligned}
& u_{n+1}(x, t)=u_{n}(x, t)+\int_{0}^{t}(\xi-t)\left(L u_{n}(x, \xi)\right. \\
& \left.+R u_{n}(x, \xi)+N u_{n}(x, \xi)-g(x, \xi)\right) d \xi \\
& =u_{n}(x, t)+\int_{0}^{t} \xi\left(L u_{n}(x, \xi)\right) d \xi-\int_{0}^{t} t\left(L u_{n}(x, \xi)\right) d \xi \\
& +\int_{0}^{t}(\xi-t)\left(R u_{n}(x, \xi)+N u_{n}(x, \xi)-g(x, \xi)\right) d \xi \\
& =u_{0}(x, t)-\int_{0}^{t}(t-\xi)\left(R u_{n}(x, \xi)\right. \\
& \left.+N u_{n}(x, \xi)-g(x, \xi)\right) d \xi .
\end{aligned}
$$

The initial values $u(x, 0)$ and $u_{x}(x, 0)$ should be used for selecting the zero-th approximation $u_{0}$, this is,

$$
u_{0}(x, t)=u_{0}(x)+t u_{1}(x) .
$$

Consequently, the exact solution may be obtained by

$$
\lim _{n \rightarrow \infty} u_{n}(x, t)=u(x, t) .
$$

\section{Solution of the perturbed NLSE (1) equation by VIM}

In this section, we outline the application of VIM to obtain explicit solution of Eq. (1) with the initial conditions $u(x, 0)=u_{0}(x), u_{x}(x, 0)=u_{1}(x)$. In the case of Kerr law nonlinearity where $F\left(|u|^{2}\right)=|u|^{2}$, the perturbed NLSE is given by

$$
\begin{aligned}
i u_{t} & +a u_{x x}+b u_{x t}+c|u|^{2} u \\
& =i\left[\alpha u_{x}+\beta\left(|u|^{2} u\right)_{x}+\gamma\left(|u|^{2}\right)_{x} u\right]
\end{aligned}
$$




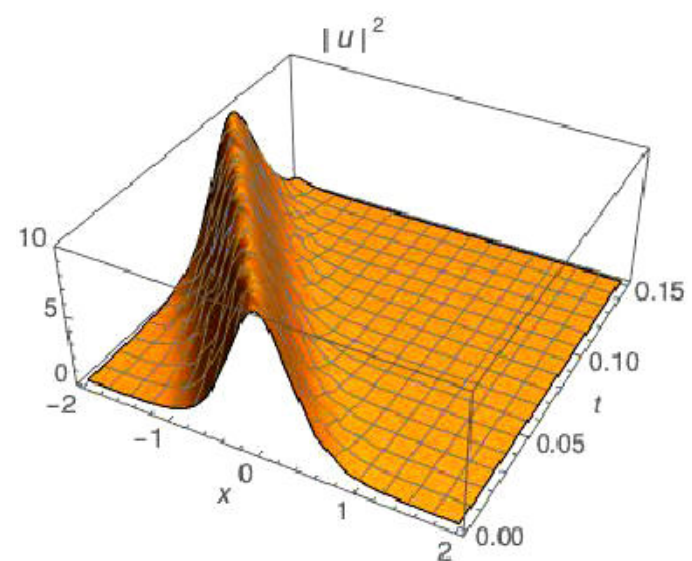

(a)

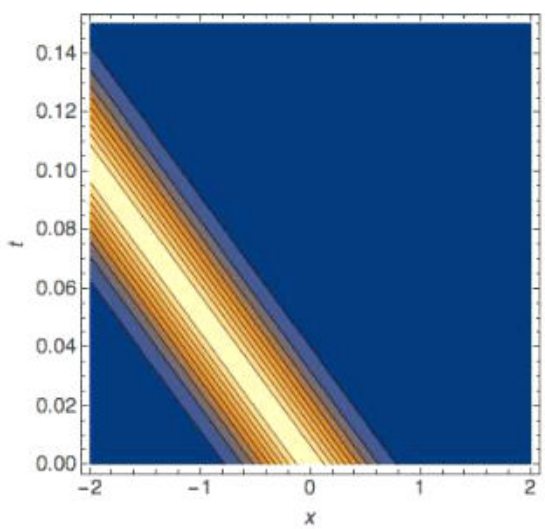

(b)

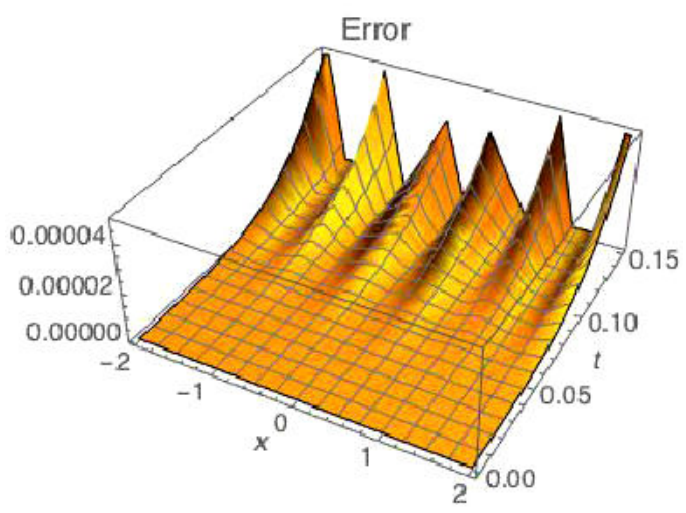

(c)

FIGURE 1. Bright solitons: Case B-1, where a) Dynamic evolution profile of $|u|^{2}$ by VIM, b) Contour plot of the wave amplitude of $|u|^{2}$, and c) Absolute error.

In the scheme of Eq. (14), we have that the linear part is $R=-\left(\alpha u_{x}+i a u_{x x}+i b u_{x t}\right)$ and the nonlinear part is $N=-i c|u|^{2} u-\beta\left(|u|^{2} u\right)_{x}-\gamma u\left(|u|^{2}\right)_{x}$.

By applying the VIM for Eq. (21) the following recurrence relation for the determination of the components $u_{n+1}(x, t)$; are obtained:

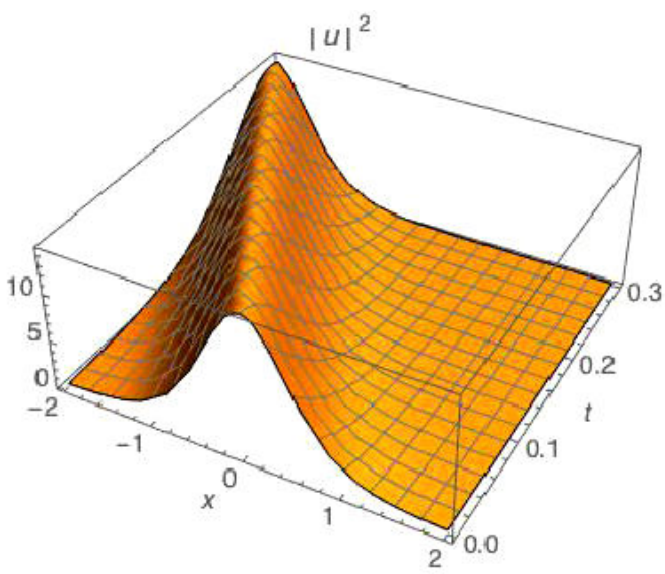

(a)

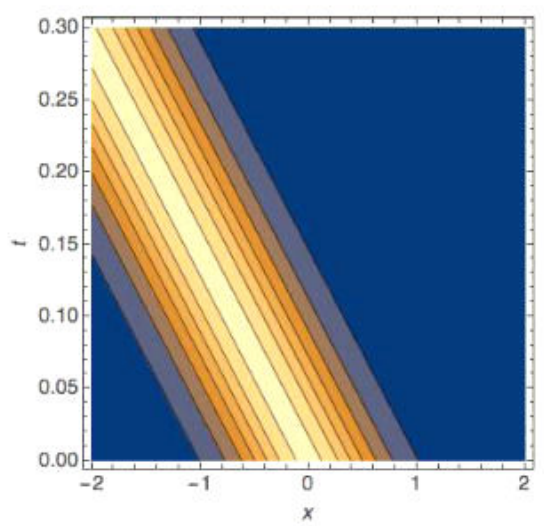

(b)

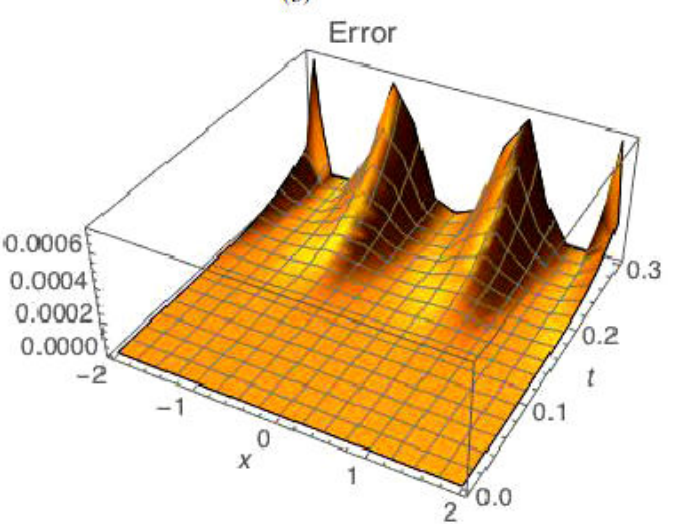

(c)

FIGURE 2. Bright solitons: Case B-2, where a) Dynamic evolution profile of $|u|^{2}$ by VIM, b) Contour plot of the wave amplitude of $|u|^{2}$, and c) Absolute error.

$$
\begin{aligned}
u_{0}(x, t) & =u_{0}(x)+t u_{1}(x), \\
u_{1}(x, t) v & =u_{0}(x, t)-\int_{0}^{t}(t-\xi) \\
& \times\left(R u_{0}(x, \xi)+N u_{0}(x, \xi)\right) d \xi,
\end{aligned}
$$




$$
\begin{aligned}
u_{2}(x, t) & =u_{0}(x, t)-\int_{0}^{t}(t-\xi) \\
& \times\left(R u_{1}(x, \xi)+N u_{1}(x, \xi)\right) d \xi, \\
u_{3}(x, t) & =u_{0}(x, t)-\int_{0}^{t}(t-\xi) \\
& \times\left(R u_{2}(x, \xi)+N u_{2}(x, \xi)\right) d \xi,
\end{aligned}
$$

and so on.

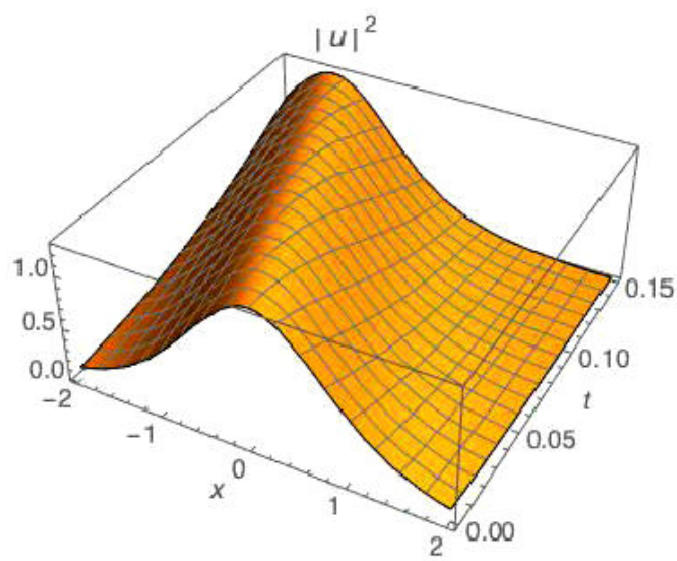

(a)

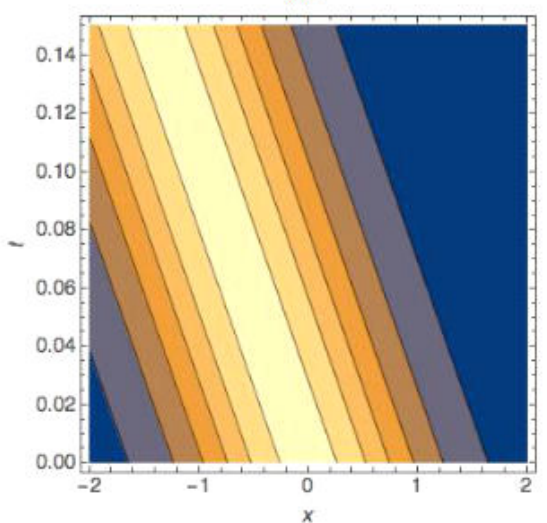

(b)

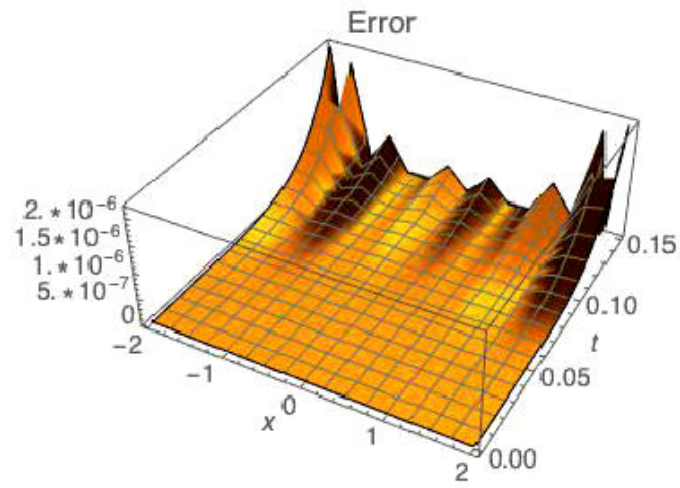

(c)

FIguRE 3. Bright solitons: Case B-3, where a) Dynamic evolution profile of $|u|^{2}$ by VIM, b) Contour plot of the wave amplitude of $|u|^{2}$, and c) Absolute error.

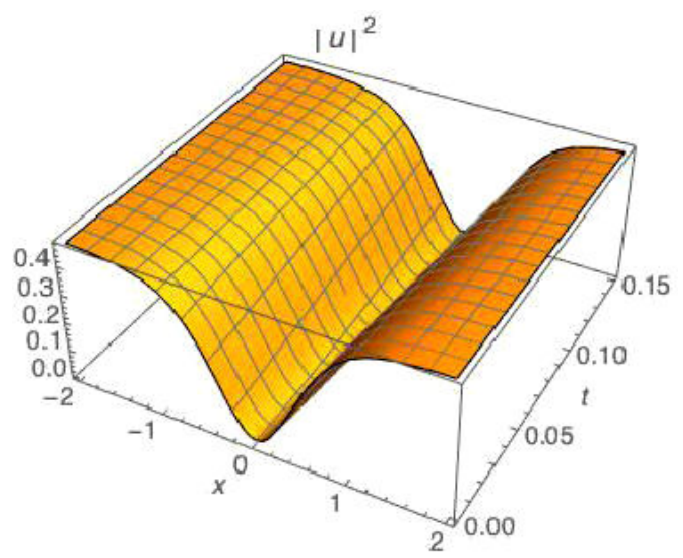

(a)

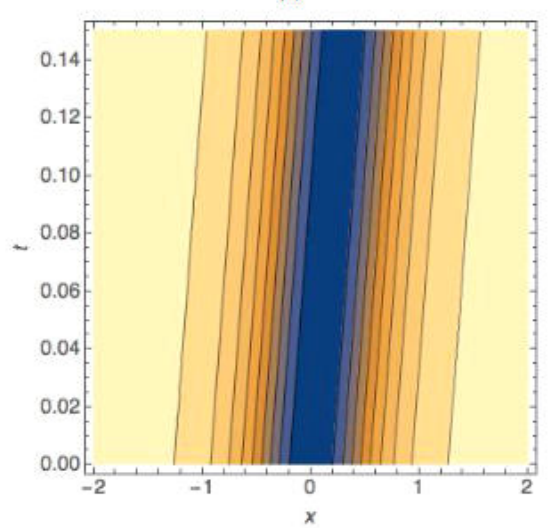

(b)

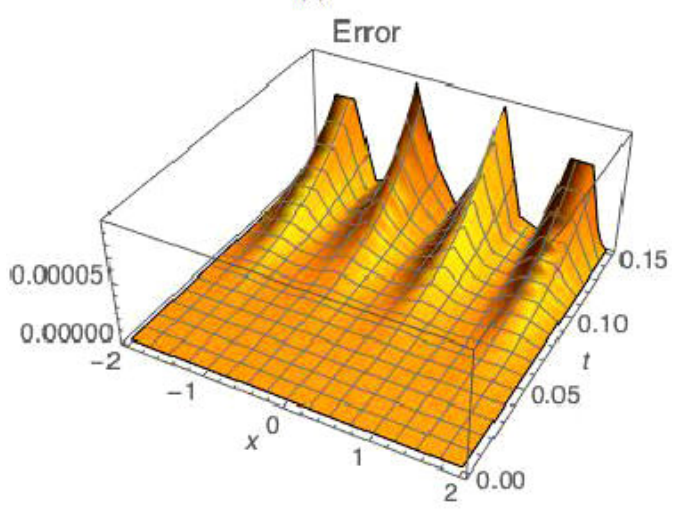

(c)

FIGURE 4. Dark solitons: Case D-1. where a)Dynamic evolution profile of $|u|^{2}$ by VIM, b) Contour plot of the wave amplitude of $|u|^{2}$, and c) Absolute error.

Continuing in this manner, the $(n+1)$-th approximation of the exact solutions for the unknown functions $u(x, t)$ can be achieved as:

$$
\begin{aligned}
u(x, t) & \approx u_{0}(x, t)+u_{1}(x, t) \\
& +u_{2}(x, t)+, \ldots,+u_{n}(x, t) .
\end{aligned}
$$




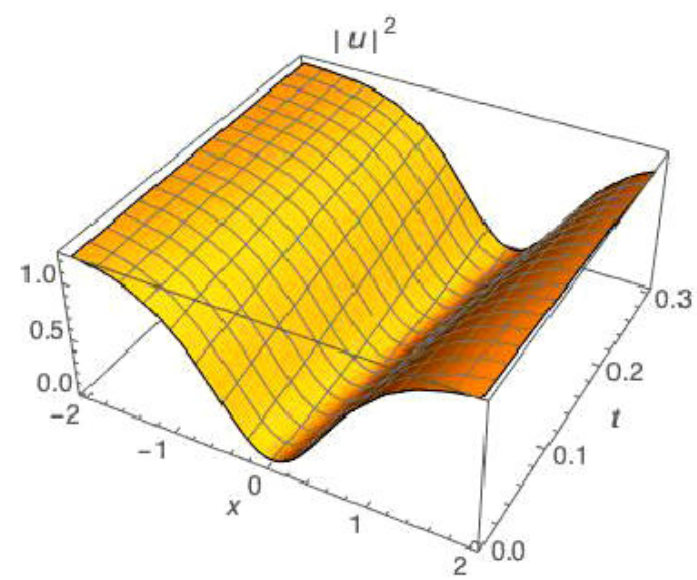

(a)

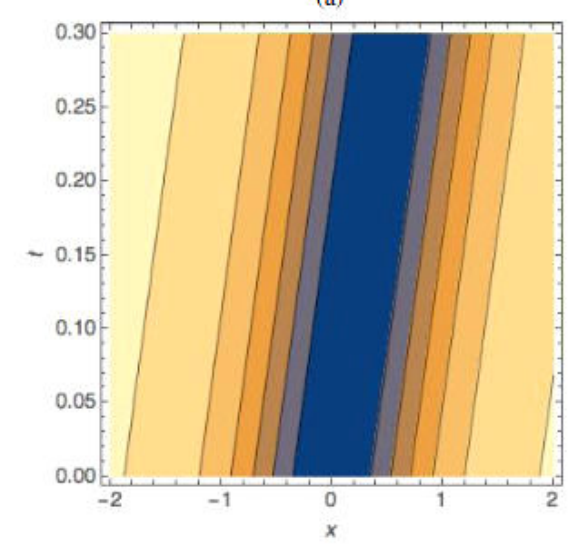

(b)

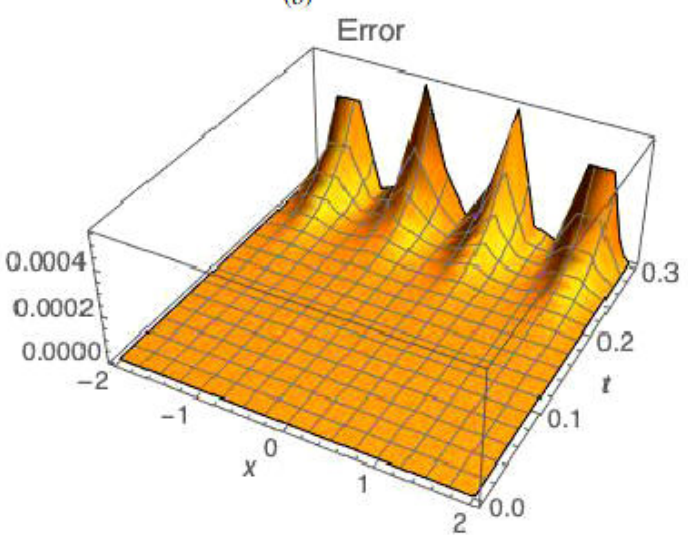

(c)

FIGURE 5. Dark solitons: Case D-2, where a)Dynamic evolution profile of $|u|^{2}$ by VIM, b)Contour plot of the wave amplitude of $|u|^{2}$, and c) Absolute error.

\section{Numerical applications and graphical results}

In this section, we implement the VIM to obtain numericanalytic solutions to the nonlinear Schrödinger equation with Kerr law nonlinearity governed by Eq. (1) and with different initial conditions. We perform numerical simulations for bright and dark optical solitions.

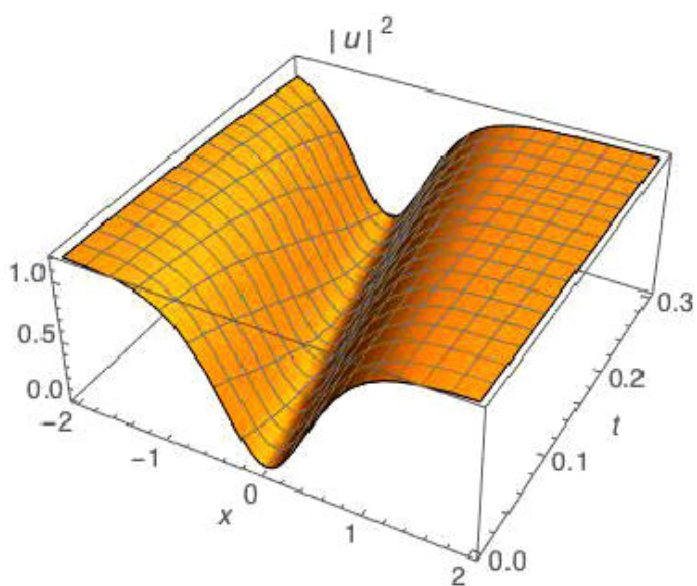

(a)

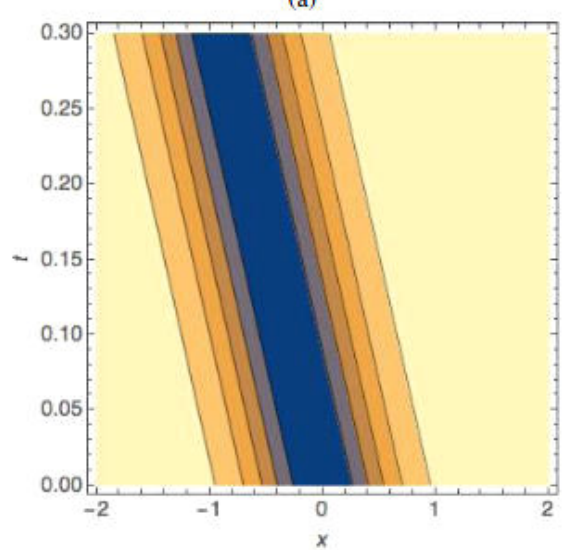

(b)

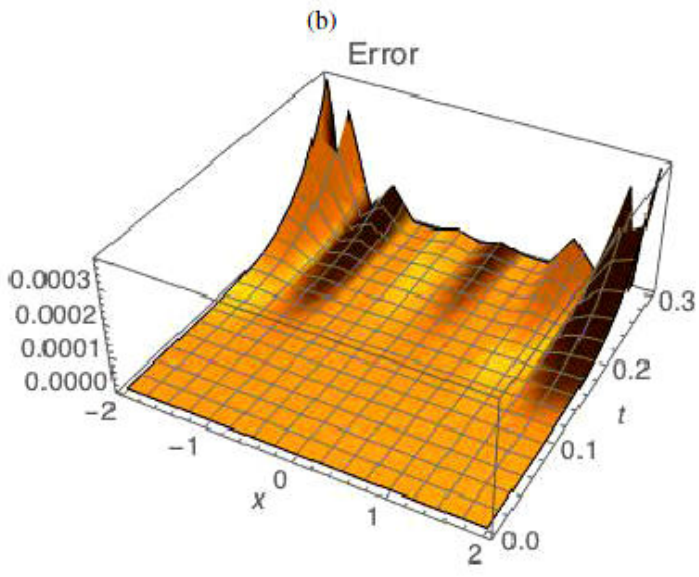

(c)

FIGURE 6. Dark solitons: Case D-3, where a) Dynamic evolution profile of $|u|^{2}$ by VIM, b) Contour plot of the wave amplitude of $|u|^{2}$, and c) Absolute error.

\section{Application to bright optical solitions}

Consider the nonlinear Schrödinger equation with Kerr law nonlinearity (21), with initial conditions

$$
\begin{aligned}
u(x, t) & =A \operatorname{sech}[B x] e^{i[-\kappa+\theta]}, \\
u_{x}(x, 0) & =-A B \operatorname{sech}[B x] \tanh [B x] e^{i[-\kappa+\theta]} .
\end{aligned}
$$


TABLE I. Bright optical solitons.

\begin{tabular}{cccccccccccccc}
\hline Cases & $a$ & $b$ & $c$ & $\alpha$ & $\beta$ & $\gamma$ & $\kappa$ & $\omega$ & $\nu$ & $A$ & $B$ & $n$ & $\mid$ Max Error $\mid$ \\
\hline$B_{1}$ & 0.1 & 3.2 & 3.8 & 1.5 & -1.0 & 1.5 & 3.0 & 0.1 & -29.5 & 3.13 & 2.32 & 14 & $1.5 \times 10^{-4}$ \\
$B_{2}$ & 0.2 & 1.5 & 4.3 & 1.3 & 1.8 & -2.7 & 1.8 & -0.7 & -6.93 & 3.81 & 1.63 & 14 & $6.0 \times 10^{-4}$ \\
$B_{3}$ & 0.4 & 1.1 & 4.2 & 1.8 & -2.1 & 3.1 & 1.3 & 0.5 & -9.29 & 1.13 & 0.96 & 14 & $1.0 \times 10^{-5}$ \\
\hline
\end{tabular}

TABLE II. Dark optical solitons.

\begin{tabular}{cccccccccccccc}
\hline Cases & $a$ & $b$ & $c$ & $\alpha$ & $\beta$ & $\gamma$ & $\kappa$ & $\omega$ & $\nu$ & $A$ & $B$ & $n$ & $\mid$ Max Error $\mid$ \\
\hline$D_{1}$ & 2.0 & 1.0 & 3.5 & 1.0 & -2.0 & 3.0 & 1.3 & 0.2 & 2.0 & 0.69 & 0.69 & 14 & $3.0 \times 10^{-4}$ \\
$D_{2}$ & 2.5 & 1.5 & 4.2 & 1.1 & -1.3 & 1.9 & 2.1 & -0.1 & 1.8 & 1.12 & 1.20 & 14 & $4.0 \times 10^{-4}$ \\
$D_{3}$ & 2.2 & 1.8 & 5.1 & 1.2 & 1.5 & -2.2 & 1.2 & 0.3 & -2.1 & 1.09 & 0.65 & 14 & $3.0 \times 10^{-4}$ \\
\hline
\end{tabular}

Table I shows the comparison between the absolute error of the exact and approximate solutions for various values of coefficients in the case of bright optical solitons. Figures 1, 2, and 3 give the plots for the approximate solutions by using VIM, contour plot of the wave amplitude of $|u|^{2}$, and absolute error, respectively.

\section{Application to dark optical solitions}

Consider the nonlinear Schrödinger equation with Kerr law nonlinearity (21), with initial conditions

$$
\begin{aligned}
u(x, t) & =A \tanh [B x] e^{i[-\kappa+\theta]}, \\
u_{x}(x, 0) & =-A B \operatorname{sech}^{2}[B x] e^{i[-\kappa+\theta]} .
\end{aligned}
$$

Table II shows the comparison between the absolute error of the exact and approximate solutions for various values of coefficientsin the case of dark optical solitons. Figures 4, 5, and 6 give the plots for the approximate solutions by using VIM, contour plot of the wave amplitude of $|u|^{2}$, and absolute error, respectively.

\section{Conclusions}

This paper addressed the dynamics of bright and dark optical solitons governed by the perturbed NLSE that appeared with
Kerr law of nonlinearity. The results are visibly meaningful with surface plots and error plots. The numerical integration scheme adopted in today's work is VIM. Thus, the integration scheme showed a grand success with the model picked for the present study. This therefore is extremely encouraging to consider other models in future such as to study the same perturbed NLSE with non-Kerr nonlinearities and to also extend the study to optical couplers, differential group delay and with DWDM topology. Such results are in the works and will be sequentially, but surely, disseminated.

\section{Acknowledgements}

The research work of the fourth author (MRB) was supported by the grant NPRP 11S-1126-170033 from QNRF and he is thankful for it.

\section{Authors contributions}

All authors contributed equally and significantly in writing this article. All authors read and approved the final manuscript.
1. W. Yu, Q. Zhou, M. Mirzazadeh, W. Liu, and A. Biswas, Phase shift, amplification, oscillation and attenuation of solitons in nonlinear optics, J. Adv. Res. 15 (2019) 69, https: //doi.org/10.1016/j.jare.2018.09.001

2. A.-M. Wazwaz, A variety of optical solitons for nonlinear Schrödinger equation with detuning term by the variational iteration method, Optik 196 (2019) 163169, https://doi. org/10.1016/j.ijleo.2019.163169

3. A.-M. Wazwaz and S. A. El-Tantawy, Optical Gaussons for nonlinear logarithmic Schrödinger equations via the variational iteration method, Optik 180 (2019) 414, https://doi.

\section{org/10.1016/j.ijleo.2018.11.114}

4. A.-M. Wazwaz and L. Kaur, Optical solitons and Peregrine solitons for nonlinear Schrödinger equation by variational iteration method, Optik 179 (2019) 804, https : / / doi .org/ $10.1016 / j . i j l e o .2018 .11 .004$

5. M. Savescu, K. R. Khan, R. W. Kohl, L. Moraru, A. Yildirim, and A. Biswas, Optical Soliton Perturbation with Improved Nonlinear Schrödinger's Equation in Nano Fibers, J. Nanoelectron. Optoelectron. 8 (2013) 208, https : // doi.org/10. $1166 /$ jno.2013.1459.

6. R. Kohl, A. Biswas, D. Milovic, and E. Zerrad, Optical 
soliton perturbation in a non-Kerr law media, Opt. Laser Technol. 40 (2008) 647, https: / / doi.org/10.1016/j. optlastec.2007.10.002

7. J.-H. He, Variational iteration method - a kind of nonlinear analytical technique: some examples, Int. J. NonLinear Mech. 34 (1999) 699, https://doi.org/10. 1016/S0020-7462(98)00048-1

8. J.-H. He and X.-H. Wu, Construction of solitary solution and compacton-like solution by variational iteration method, Chaos
Solitons Fractals 29 (2006) 108, https://doi.org/10. $1016 / j$.chaos.2005.10.100

9. S. Momani and S. Abuasad, Application of He's variational iteration method to Helmholtz equation, Chaos Solitons Fractals 27 (2006) 1119, https://doi.org/10.1016/j. chaos.2005.04.113

10. A.-M. Wazwaz, The variational iteration method for rational solutions for $\mathrm{KdV}, K(2,2)$, Burgers, and cubic Boussinesq equations, J. Comput. Appl. Math. 207 (2007) 18, https: //doi.org/10.1016/j.cam.2006.07.010 